\title{
Implementing Gene Expression Programming in the Parallel Environment for Big Datasets' Classification
}

\author{
Joanna Jȩdrzejowicz \\ Institute of Informatics \\ Faculty of Mathematics, Physics and Informatics \\ University of Gdańsk, 80-308 Gdańsk, Poland \\ Piotr Jȩdrzejowicz and Izabela Wierzbowska* \\ Department of Information Systems \\ Gdynia Maritime University \\ Morska 81-83, 81-225 Gdynia, Poland \\ *i.wierzbowska@wpit.umg.edu.pl \\ Received 31 December 2018 \\ Revised 25 February 2019 \\ Accepted 4 March 2019 \\ Published 26 April 2019
}

\begin{abstract}
The paper investigates a Gene Expression Programming (GEP)-based ensemble classifier constructed using the stacked generalization concept. The classifier has been implemented with a view to enable parallel processing with the use of Spark and SWIM - an open source genetic programming library. The classifier has been validated in computational experiments carried out on benchmark datasets. Also, it has been inbvestigated how the results are influenced by some settings. The paper is an extension of a previous paper of the authors.
\end{abstract}

Keywords: Gene expression; classification; big data.

\section{Introduction}

The classification is one of the problems in data mining and its task is to predict the class for previously unseen data after learning patterns or relationships from data with known classes. The classification algorithms use various models, such as decision trees, neural network, statistical or clustering techniques. In the recent years, Gene Expression Programming (GEP) has also been used as an effective classification algorithm.

*Corresponding author.

This is an Open Access article published by World Scientific Publishing Company. It is distributed under the terms of the Creative Commons Attribution 4.0 (CC-BY) License. Further distribution of this work is permitted, provided the original work is properly cited. 
GEP was introduced by Ferreira. ${ }^{1}$ It is an evolutionary algorithm in which genes in the evolving population represent programs, and their fitness corresponds to how well the programs calculate or perform the given task.

GEP is a time-consuming algorithm with many iterations and parameters. There have been many attempts to improve its performance, especially for data characterized by massive amount. For example Liu et al. ${ }^{2}$ considered the parallelizing GEP algorithm to enable large-scale classification, using majority-voting to combine a number of GEP-based classifiers obtained for separate data chunks.

Jȩdrzejowicz and Jȩdrzejowicz in a series of papers ${ }^{3-6}$ proposed several approaches to combining expression trees induced by GEP applied to random subsets of the original data.

In Ref. 6, the authors proposed a GEP-based batch ensemble classifier that uses stacked generalization concept, in which the higher-level model is created from lower level classifiers in the form of a meta-gene. Later, in Ref. 7, the model was implemented with a view to enable parallel processing with the use of Spark - an open source data processing engine and SWIM - an open source library that implements, in multi-threaded way, the functionality of GEP.

This paper is an extension of Ref. 7, with the use of the same model, however, the paper also investigates how predictive accuracy may be influenced by such elements of the model like grammar or number of classifiers in batch.

The paper is organized as follows: In Sec. 2, the related work is briefly reviewed. In Sec. 3, the description of the classifier is recalled, both for binary (Sec. 3.1) and multiclass (Sec. 3.2) datasets. In Sec. 4, the details of the implementation are discussed. Section 5 presents the experiment results in which the classifier has been used. We also show how the results are influenced by some of the algorithm settings. Section 6 gives a summary of the work presented in this paper and proposes directions of future research.

\section{Related Work}

In her book, Ferreira ${ }^{8}$ shows how to evolve basic GEP classification algorithms and applies them to a simple dataset. An important extension of the above idea was suggested by Zhou et al. ${ }^{9}$ They proposed to use different combinations of attributes and to enrich the fitness function through considering two criteria - gains in rule consistency and rule completeness. Wang et al. ${ }^{10}$ proposed an extension to the basic GEP. Authors claim that basic GEP classifiers could be improved through removing irrelevant fragments from classification rules.

Weinert and Lopes ${ }^{11}$ proposed their own method for encoding rules together with the set of genetic operators assuring rule integrity. Next, they designed and implemented the classifier system called GEPCLASS producing flexible classification rules. Duan et al. ${ }^{12}$ proposed another extension to the basic GEP. The approach introduced dynamically controlled classification threshold and was named Distance Guided Evolution Algorithm. Further modification of the basic GEP was suggested 
by Liu et $a l .^{13}$ The authors proposed using GEP for inducing fuzzy classification rules. Usual mathematical operators were replaced by logical ones to calculate the chromosome quality. An effective extension of the basic GEP classiffier named MCGEP was recently proposed by Guerrero-Enamorado et al. ${ }^{14}$ The proposed classifier uses a Michigan approach where each individual encodes a single classification rule. Evolution process is based on elitism and the redundant individuals are penalized.

As it has been observed by Xu et al. ${ }^{15}$ in big data research, GEP encounters low efficiency issue due to its long time mining processes. To improve the efficiency of GEP, their paper proposes a parallelized GEP algorithm using MapReduce computing model. Significant improvement of the basic GEP classifiers can be obtained through combining GEP-evolved weak classifiers into a classifier ensemble. In Ref. 16, two well-known ensemble techniques - bagging and boosting - were used to enhance the generalization ability of GEP classifiers. Different approach to building GEP-based classifier ensembles was proposed by $\mathrm{Wu}$ et al. ${ }^{17}$ The idea was to construct weak classifiers from different subsets of attributes controlling the diversity among these subsets through applying a variant of niching technique.

\section{GEP Ensemble Based on Stack Generalization Concept}

GEP is an evolutionary algorithm in which genes are programs. While trained on data with known classes, the genes in the population evolve, so that they can serve as classifier for the given data. The genes are evaluated according to how well they realize their required functionality — which in this case means how big percentage of the data they can classify well.

The algorithms in this section originate from Ref. 6 .

\subsection{Binary classification}

If only two classes exist for data, the task of classification of the data is called binary classification. In binary classification, each gene $g$ is an expression that for a given data instance, $r$ produces result $g(r)$, and the result $g(r)$ equals true or false, depending on whether the instance belongs or not to one class, chosen from the two classes.

The structure of genes is described by the grammar presented in Fig. 1. In this grammar,

- there is a number of rules that compare some constant - a random values defined as const - with expression built from the values of attributes;

- there is a number of rules that compare two expressions built on the attributes;

- above rules may be used to build more complex boolean expressions with the use of boolen operators.

The leaves in the grammar are the following:

- DataFeature Value stands for a leaf, that is a random value chosen from the values appearing in the sample of the data currently kept in the algorithm memory. 


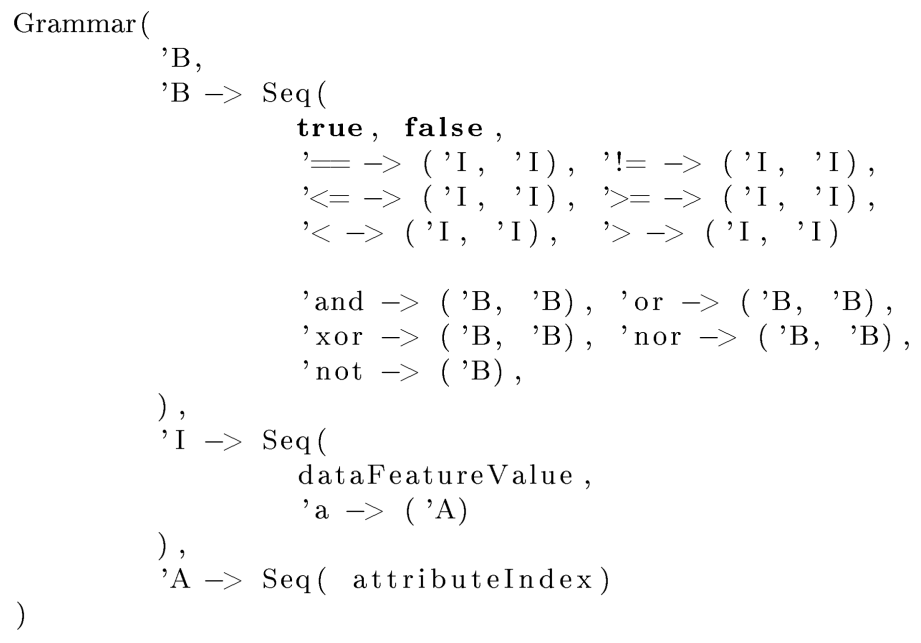

Fig. 1. Grammar describing genes.

- AttributeIndex stands for random attribute index. Thus, $a(0)$ in a gene equals the value of the first feature in the instance of data $a$.

The grammar in Fig. 1 is defined in Scala, in accordance with how syntactically correct programs are defined in the SWIM library, which was used for the implementation. The definition is a copy from the source code. In Scala, 'B denotes symbol $\mathrm{B}$, Seq stands for sequence, values in brackets create a tuple (a sequence of values of multiple types). Notation 'symbol $->$ value binds 'symbol with value (in another tuple).

For classification of a dataset, the process of training is carried out $n g$ times. In this way, a population $P G$ of $n g$ base classifiers (genes) is created. The classifiers from $P G$ serve as the base for the next step - training an ensemble classifier (or metagene), which uses genes from $P G$ and which best classifies training data.

Meta-genes are described by the grammar from Fig. 2. In the grammar, GeneIndex stands for the index of a gene in $P G$.

To evaluate genes (or meta-genes), they are applied to each instance of data in the training set, and the number of instances for which the gene (meta-gene) returned wrong class is calculated. In the process of evolution, the number is minimalized.

Binary classification with meta-genes is described in Algorithm 1.

\subsection{Multi-class classification}

In classifications of the data with more than two classes, binary expressions, as defined in binary classification, are also used. For each class, a binary classifier is constructed, such that it can distinguish the instances of the given class from all instances of other classes. Later, when such binary classifiers are available for each class, they are used jointly, as described as follows. 


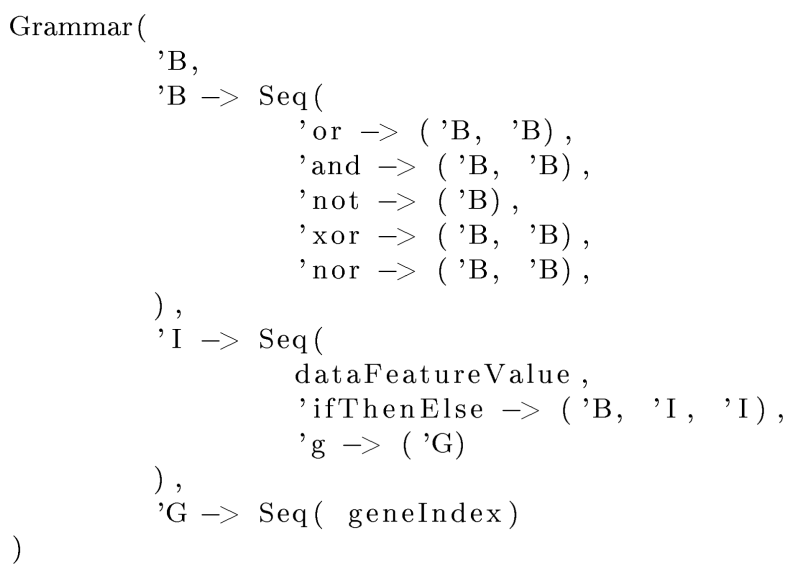

Fig. 2. Grammar describing meta-genes.

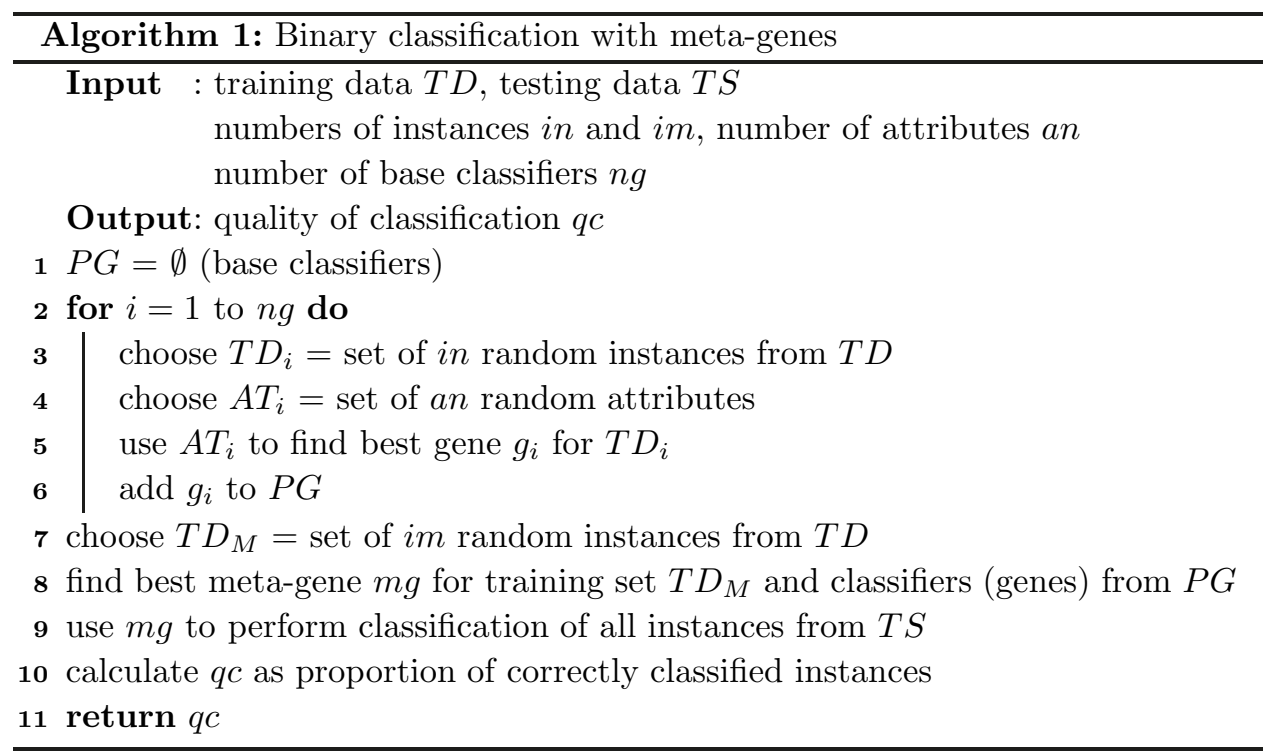

In the process of learning, a set $P G$ of $n g$ binary classifiers is generated for each class. The details of how the set is created are shown in Algorithm 2.

The classifiers are used to determine the class of a row of data in the process of voting: each classifier votes for or against the class that it has learnt to recognize, where vote against a class is equivalent to voting for each of the other classes. After summing up all the votes, the class with the maximum number votes is chosen as the result of the multi-class classification. The process is 3 . 

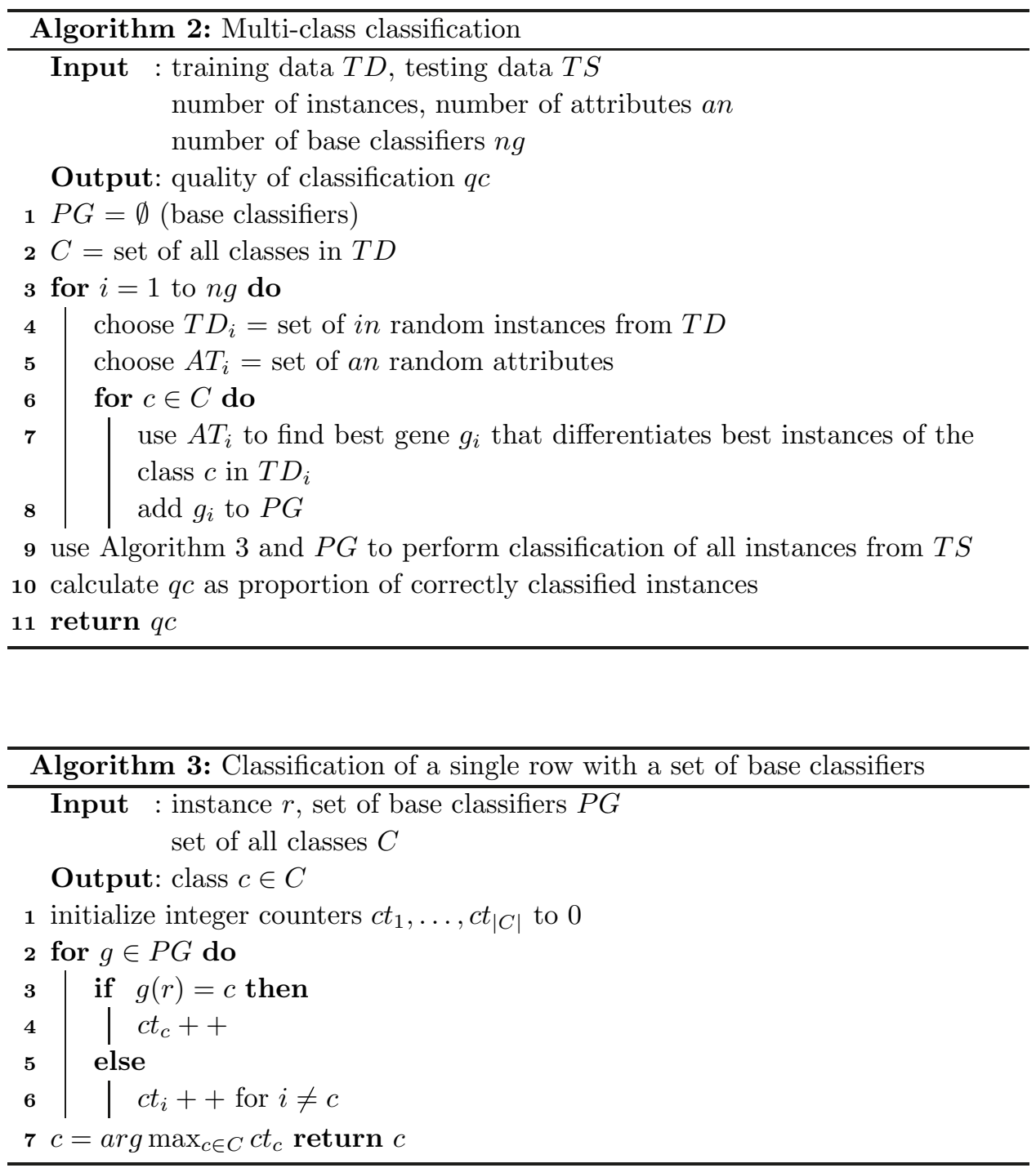

\section{Implementation}

The classifier was implemented in Scala with the use of SWIM ${ }^{18}$ and Apache Spark ${ }^{19}$ libraries.

SWIM is a Scala library that implements basic functionalities of Genetic Programming. To realize the functionality of evolutionary algorithm, SWIM relies on the FUEL library, which implements the evolutionary computation workflow in Scala, and originated in Ref. 20. The SWIM library has been modified to allow the elitism in the genetic algorithm. 
SWIM allows to define genes (programs) syntax by defining a grammar and a matching semantics. The grammars described in Sec. 3.1 (Figs. 1 and 2) are both grammars as they were used in Scala with SWIM.

Apart of the grammars, SWIM requires defining specific program's evaluation (fitness functions for genes), which in the case of classification has to count the percentage of correctly classified rows of data.

Both SWIM and FUEL support sequential and parallel evaluation of candidate solutions, depending on the value of one of the parameters. Also, even if Spark is used only in local mode, like in the following experiments, it allows for some number of parallel threads to be executed. Thus, the implementation of the classifier enables three sources of parallelism in a single run of the algorithm:

- parallel evaluation of candidate solutions in GEP, as supported by SWIM and FUEL;

- parallel creation of $n g$ base classifiers, as supported by Scala Parallel Collection;

- parallel threads in Spark, as supported by a number of Spark workers.

\section{Experiment}

A series of experiments has been run to assess the accuracy and running time of the implementation. All experiments were carried out on one standalone Spark equipped environment with 16GB RAM and Core i7-3630QM processor.

The experiments with the classifier have been carried on the datasets and are shown in Table 1. The datasets in the table have been taken from $\mathrm{UCI}^{27}$ and OpenML ${ }^{28}$ repositories. The table contains name, number of instances and number of attributes of each dataset. Also, there are some references to the literature, which reported some classification results for the data.

Classification results are presented in Table 2 and are similar to the ones presented in Ref. 6, though it may be seen that the running time was longer than in Ref. 6 , in which every experiment took less then $1 \mathrm{~min}$.

To assess how introducing concurrency affects the computation time, the program has been run with different settings defining parallel (or sequential) execution:

- parallel or sequential evaluation within GEP,

- parallel or sequential calculation of $n g$ base classifiers,

Table 1. Datasets used in the reported experiment.

\begin{tabular}{lrcccc}
\hline Dataset & Instances & Attributes & Classes & $\begin{array}{c}\text { Literature reported } \\
\text { accuracy }\end{array}$ & References \\
\hline Airlines & 539,383 & 8 & 2 & 0.663 & 21 \\
Credit Card & 30,000 & 24 & 2 & 0.822 & 22 \\
Wilt & 4,839 & 6 & 2 & 0.946 & 23 \\
Banknote Authentication & 1,372 & 5 & 2 & 0.959 & 24 \\
Bank Marketing & 45,211 & 17 & 2 & 0.844 & 25 \\
KDD Cup & $4,000,000$ & 42 & 2 & 0.938 & 26 \\
\hline
\end{tabular}


Table 2. Experiments results.

\begin{tabular}{lcccccc}
\hline Dataset & Accuracy & $+/-$ & \% of instances & $\begin{array}{c}\text { Number of } \\
\text { base classifiers }\end{array}$ & Time (s) & Accuracy in Ref. 6 \\
\hline Airlines & 0.626 & 0.03 & 0.001 & 10 & 169 & \\
Credit Card & 0.813 & 0.01 & 0.01 & 10 & 261 & 0.8154 \\
Wilt & 0.946 & 0.01 & 0.1 & 10 & 184 & 0.9431 \\
KDD Cup & 0.943 & 0.02 & 0.00001 & 3 & 4848 & 0.8083 \\
& & & & & & (for KDD Cup 10\%) \\
\hline
\end{tabular}

- parallel or sequential repetitions of the 10-cross-validation scheme,

- one or more threads in Spark.

The results are shown in Figs. 3 and 4, showing the computations time, in s, for two datasets, Credit Card and Airlines, with 30, 000 and 539, 383 instances and sample of the size of 300 and 540 instances, respectively, for all 10 repetitions of the 10 -crossvalidation scheme.

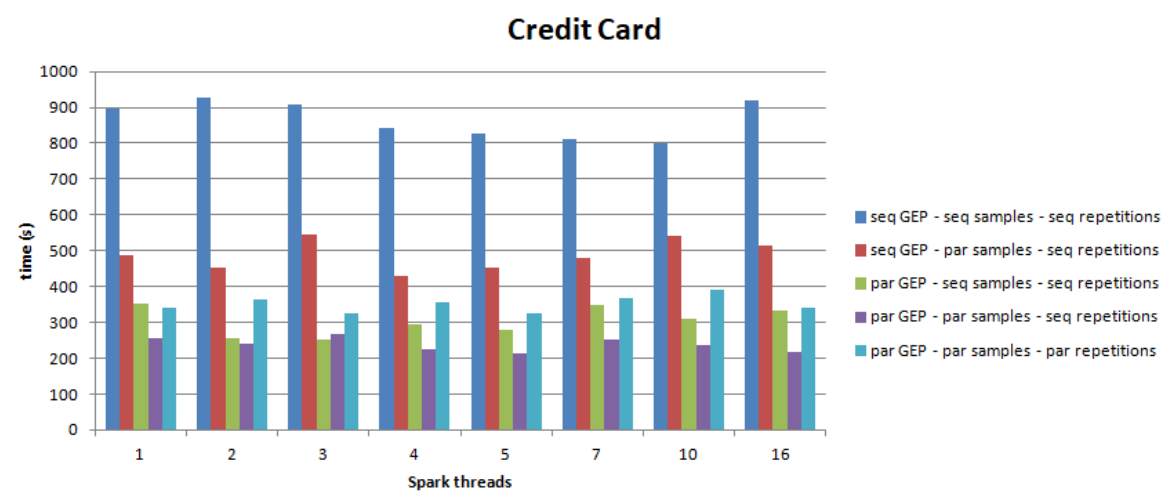

Fig. 3. Time (in s) of 10 repetitions of cross-validation classification for Credit Card dataset.

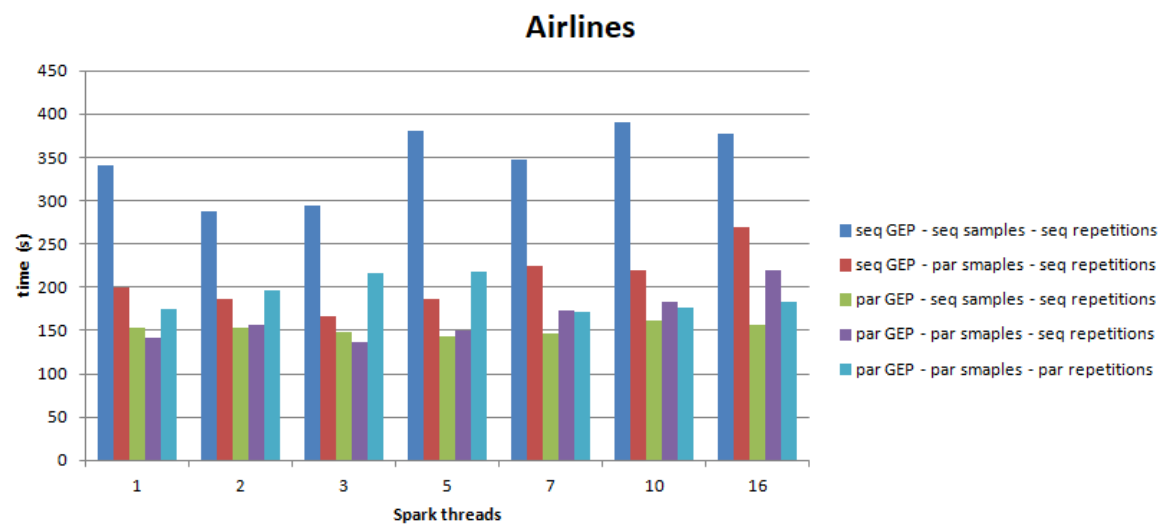

Fig. 4. Time (in s) of 10 repetitions of cross-validation classification for Airlines dataset. 
As it may be seen, the computation time depends mostly on how GEP is run with or without concurrency in the process of searching for best programs (genes). Relatively small impact of the number of threads used in Spark may be connected with the fact that Spark is used only for sampling the dataset and - after the classification program has been found - for the classification of the testing data. This is definitely less time-consuming than GEP. It is worth to remind that all calculations are done on a single node, thus it may be the reason why setting all possible processes parallel results in longer time of running — in this case, the limits of how much concurrency is handled may have been exceeded.

\subsection{The number of base classifiers}

In Algorithm 1, each meta-gene itself is a classifier that may be used alone for the task of classification. Increasing the number of classifiers may increase the accuracy, but it also requires longer time. It may be observed in Fig. 5 which presents running times and accuracies for different numbers of base classifiers in the case of Banknote Authentication dataset. In this case, it can also be noted that at some point, increasing the number of meta-genes does not affect the result and only renders longer computation time.

\subsection{The grammar used to define genes}

The grammar that is used to define genes may be extended with some additional functions or expressions. In Fig. 6, there is an example of a grammar in which

- a number of trigonometric and math functions have been added: sin, cos, abs, + , - , min, $\max$

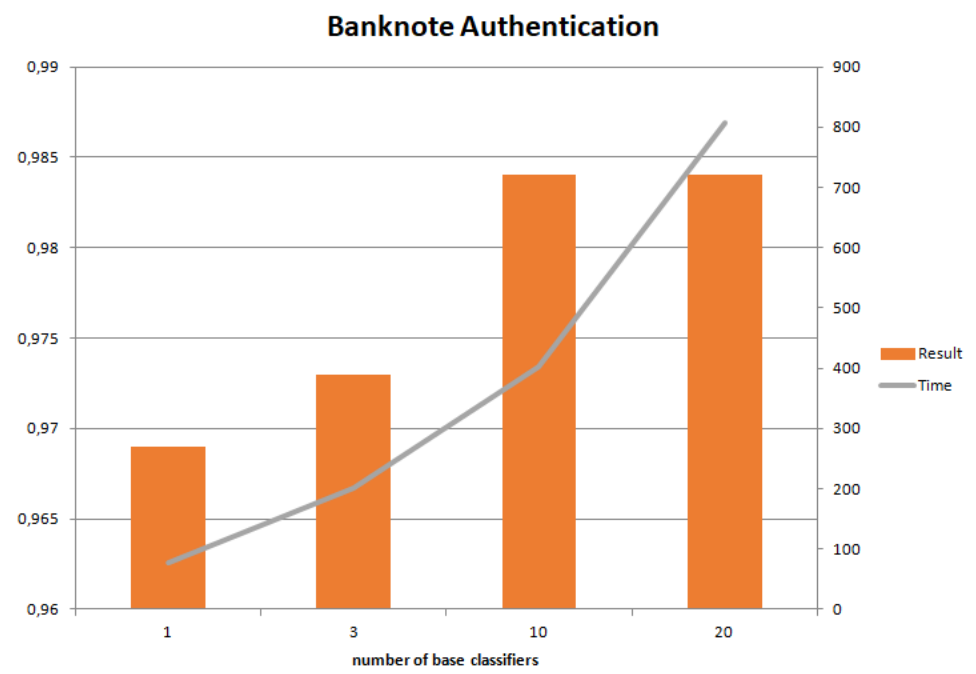

Fig. 5. Number of base classifiers in Banknote Authentication. 


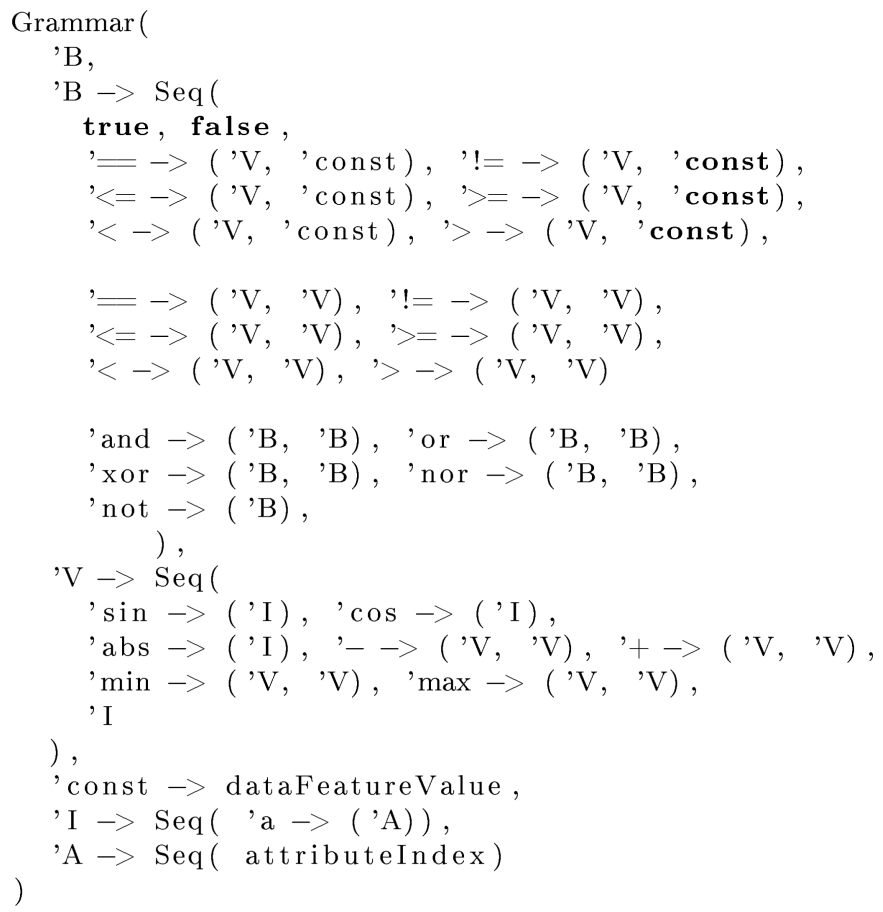

Fig. 6. Modified grammar describing genes in binary classification.

- the grammar has been written in such a way that constants may be used only as a part of comparison with some expression that uses functions (the constants are never used as arguments of functions in order to not unnecessarily increase the length of genes).

The grammar has been used in an experiment in which the results of classification are shown depending on which functions are used to define classifier. In this experiment, specific functions were or were not used in the process of classification, and the results were compared.

The results obtained for Banknote Authentication dataset are shown in Fig. 7. It may be seen that the choice of functions may significantly influence the results.

It seems to be obvious that the impact depends on the task. Similar experiment on Bank Marketing or Wilt datasets resulted in achieving constant accuracies, not influenced by which set of functions has been used (though again using more functons relulted in longer computation time).

\subsection{Sampling vs processing all data}

In Refs. 2 and 15, GEP algorithm and MapReduce were used for the classification of data. The idea was to split the training dataset into a number of subsets, and for each subset run GEP to receive a classifier. Due to the use of MapReduce, the classifiers 


\section{Banknote Authentication}

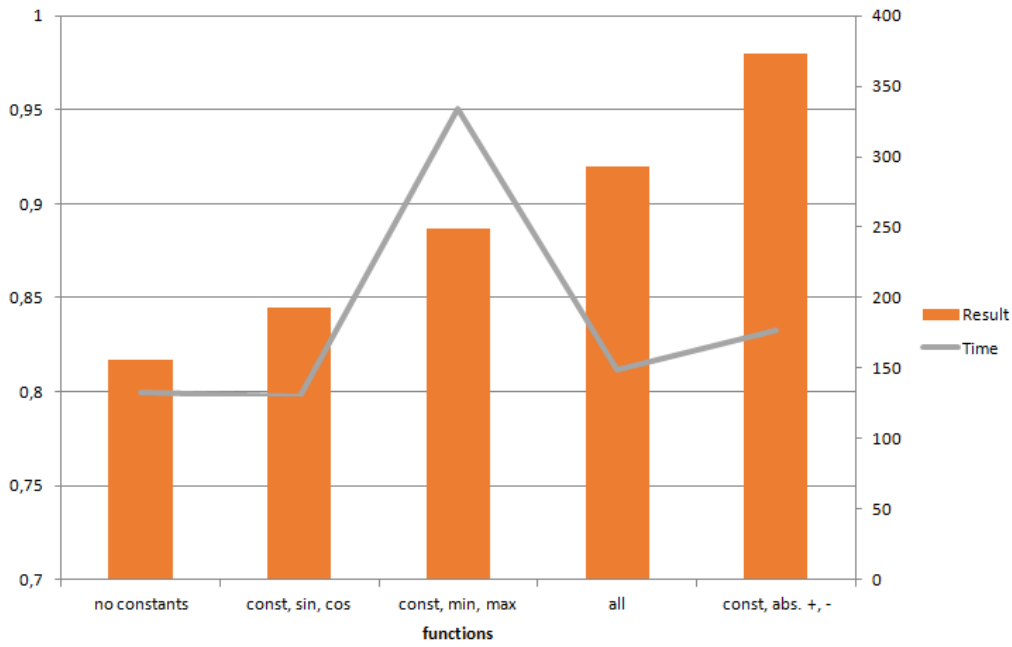

Fig. 7. Results obtained for different functions used in the grammar describing Genes and Banknote Authentication dataset.

could be created in parallel, significantly reducing the running time of the algorithm. For the obtained classifiers, the ensemble techniques with bootstraping and majority voting were adopted.

In both papers, the authors carried out one experiment with the data of significant size to assess executing time of the proposed algorithms. They used small Wine dataset with 178 instances, 13 attributes and 3 classes. In order to increase the size of the data, the dataset was artificially inflated to reach the size of 1,024 MB.

In Ref. 15, 60 instances from the original Wine dataset have been used as the testing set, the rest was multiplied to $1,024 \mathrm{MB}$ and was used as the training data. A parallel GEP-based algorithms classified data in about 5,000 s. The experiment was run on a cluster with five nodes. In the experiment, the authors did not provide accuracy, however the best accuracy for the original dataset was about $90 \%$.

Algorithm 2 was run on data prepared in the same way. It used a single sample of the size $0.0015 \%$ and a standalone Spark-equipped environment. It produced classification results with accuracy $92 \%$ in $556 \mathrm{~s}$ using only the single node.

Thus, it may be unnecessary to use the whole dataset for creating the classifiers. Sampling may result in similar quality in significantly shorter time and using only a single node.

\section{Conclusion}

The paper presents an implementation of an algorithm described in Ref. 6 . The algorithm uses Apache Spark, and is implemented with a view to use some parallelism. For GEP, the SWIM library ${ }^{18}$ is used. 
The implementation produces results similar to those in Ref. 6, though it needs slightly longer time. However, since it uses Apache Spark for accessing the data, it is not limited by the size of the dataset or its location — in the case of distributed data.

The paper also investigates how the result is influenced by some setting: for example, the number of meta-genes or the choice of the grammar that defines genes. In both cases, the accuracy may increase (up to a point) with the use of more metagenes or more complicated grammar, however, in such cases, obtaining the classifier requires more time.

The solution in which the classifiers are created from samples of data is compared to similar solution with many GEP-based classifiers that operate on the whole dataset, splitted to smaller data chunks. In the case of sampling, the accuracy is similar, but the algorithm is much faster.

Future research will focus on investigation how much more powerful computational environment can be effectively used with a view to increase the accuracy of classification and to upgrade the speed-up factor. We also intend to evaluate various possible ensemble architectures.

\section{References}

1. C. Ferreira, Gene expression programming: A new adaptive algorithm for solving problems, Complex Syst. 13(2) (2001) 87-129.

2. Y. Liu, C. Ma, L. Xu, X. Shen, M. Li and P. Li, Mapreduce-based parallel gep algorithm for efficient function mining in big data applications, Concurrency Comput. Pract. Exp. 30(23) (2018) e4379.

3. J. Jẹdrzejowicz and P. Jȩdrzejowicz, Gep-induced expression trees as weak classifiers, in Proc. 8th Industrial Conf. Advances in Data Mining: Medical Applications, E-Commerce, Marketing, and Theoretical Aspects, ICDM '08 (Springer-Verlag, Berlin, Heidelberg, 2008), pp. 129-141.

4. J. Jędrzejowicz and P. Jędrzejowicz, A family of gep-induced ensemble classifiers, in Proc. 1st Int. Conf. Computational Collective Intelligence. Semantic Web, Social Networks and Multiagent Systems, ICCCI '09 (Springer-Verlag, Berlin, Heidelberg, 2009), pp. 641-652.

5. J. Jędrzejowicz and P. Jȩdrzejowicz, Combining expression trees, in 2013 IEEE Int. Conf. Cybernetics (CYBCO) (IEEE, New York, 2013), pp. 80-85.

6. J. Jȩdrzejowicz and P. Jȩdrzejowicz, Gene expression programming ensemble for classifying big datasets, in 9th Int. Conf. Computational Collective Intelligence ICCCI 2017, Nicosia, Cyprus, 27-29 September 2017, Proc. Part II, eds. N. T. Nguyen, G. A. Papadopoulos, P. Jȩdrzejowicz, B. Trawiński and G. Vossen (Springer International Publishing, Cham, 2017), pp. 3-12.

7. J. Jȩdrzejowicz, P. Jȩdrzejowicz and I. Wierzbowska, Parallel gep ensemble for classifying big datasets in Int. Conf. Computational Collective Intelligence (Springer, Cham, 2018), pp. $234-242$.

8. C. Ferreira, Gene Expression Programming: Mathematical Modeling by an Artificial Intelligence (Springer, Berlin, Heidelberg, 2006).

9. C. Zhou, W. Xiao, T. M. Tirpak and P. C. Nelson, Evolving accurate and compact classification rules with gene expression programming, IEEE Trans. Evol. Comput. 7 (2003) 519-531. 
10. W. Wang, Q. Li and Z. Cai, Finding compact classification rules with parsimonious gene expression programming, in 2005 Int. Conf. Neural Networks and Brain (IEEE, New York, 2005), pp. 702-705.

11. W. R. Weinert and H. S. Lopes, Gepclass: A classification rule discovery tool using gene expression programming, in Advanced Data Mining and Applications, eds. X. Li, O. R. Zaïane and Z. Li (Springer, Berlin, Heidelberg, 2006), pp. 871-880.

12. L. Duan, C. Tang, T. Zhang, D. Wei and H. Zhang, Distance guided classification with gene expression programming, in Advanced Data Mining and Applications, eds. X. Li, O. R. Zaïane and Z. Li (Springer, Berlin, 2006), pp. 239-246.

13. X. Liu, Z. Cai and W. Gong, An improved gene expression programming for fuzzy classification, in Advances in Computation and Intelligence, eds. L. Kang, Z. Cai, X. Yan and Y. Liu (Springer, Berlin, Heidelberg, 2008), pp. 520-529.

14. A. Guerrero-Enamorado, C. Morell, A. Y. Noaman and S. Ventura, An algorithm evaluation for discovering classification rules with gene expression programming, Int. J. Comput. Intell. Syst. 9(2) (2016) 263-280.

15. L. Xu, Y. Huang, X. Shen and Y. Liu, Parallelizing gene expression programming algorithm in enabling large-scale classification, Sci. Program. 2017 (2017) 10pp.

16. Q. Li, W. Wang, S. Han and J. Li, Evolving classifier ensemble with gene expression programming, in Proc. Third Int. Conf. Natural Computation - Volume 03, ICNC '07 (IEEE Computer Society, Washington, DC, 2007), pp. 546-550.

17. J. Wu, C. Tang, J. Zhu, T. Li, L. Duan, C. Li and L. Dai, An attribute-oriented ensemble classifier based on niche gene expression programming, in Third Int. Conf. Natural Computation (ICNC 2007) (IEEE, New York, 2007), pp. 525-529.

18. Swim library http://github.com/kkrawiec/swim, Accessed on 30 September 2017.

19. Apache spark, http://spark.apache.org/, Accessed on 30 September 2017.

20. K. Krawiec, Behavioral Program Synthesis with Genetic Programming, Studies in Computational Intelligence, Vol. 618 (Springer International Publishing, Berlin, 2016), 172pp, http://www.cs.put.poznan.pl/kkrawiec/bps.

21. X. Limón, A. Guerra-Hernández, N. Cruz-Ramírez, H.-G. Acosta-Mesa and F. Grimaldo, A windowing strategy for distributed data mining optimized through gpus, Pattern Recognit. Lett. 93(11) (2016) 29-30.

22. S. Neema and B. Soibam, The comparison of machine learning methods to achieve most cost-effective prediction for credit card default, J. Manag. Sci. Bus. Intell. 2(8) (2017) 36-41.

23. A. Jalalirad and T. Tjalkens, Using feature-based models with complexity penalization for selecting features, J. Signal Process. Syst. 90 (2018) 201-210.

24. A. Ghazvini, J. Awwalu and A. A. Bakar, Comparative analysis of algorithms in supervised classification: A case study of bank notes dataset, Int. J. Comput. Trends Technol. 17(1) (2014) 39-43.

25. A. Koç and Z. Yeniay, A comparative study of artificial neural networks and logistic regression for classification of marketing campaign results, Math. Comput. Appl. 18 (2013) 392-398.

26. M. Almseidin, M. Alzubi, S. Kovacs and M. Alkasassbeh, Evaluation of machine learning algorithms for intrusion detection system, in 2017 IEEE 15th Int. Symp. Intelligent Systems and Informatics (SISY) (IEEE, New York, 2017), pp. 000277-000282.

27. K. Bache and M. Lichman, UCI machine learning repository, UCI Machine Learning Repository University of California, Irvine, School of Information and Computer Sciences (2013).

28. J. Vanschoren, J. N. van Rijn, B. Bischl and L. Torgo, OpenML: networked science in machine learning, SIGKDD Explorations, Vol. 15, No. 3 (ACM, New York, NY, USA, 2017), pp. 49-60. 